\title{
Domains of initiator tRNA and initiation codon crucial for initiator tRNA selection by Escherichia coli IF3
}

\author{
Dieter Hartz, Jonathan Binkley, Tamy Hollingsworth, and Larry Gold ${ }^{1}$ \\ Department of Molecular, Cellular, and Developmental Biology, University of Colorado, Boulder, Colorado 80309 USA
}

\begin{abstract}
Initiation factors are used by Escherichia coli to select the initiator tRNA over elongator tRNAs during translation initiation. IF3 appears to "inspect" the anticodon end of the tRNA, probably along with the initiation codon. The anticodon stem and loop of the initiator tRNA, together with part of the initiation codon of the mRNA, can be thought of as a unit. Changes made in the anticodon stem, the anticodon loop, or the anticodon of an initiator tRNA fragment result in a loss of selection by IF3 in an in vitro assay for translation initiation. IF3 allows the selection of an initiator tRNA anticodon stem and loop fragment on GUG and UUG codons but does not select that tRNA fragment in response to AUU.
\end{abstract}

[Key Words: Translation; initiation; IF3; tRNA]

Received April 12, 1990; revised version accepted July 17, 1990.

Escherichia coli initiation factor IF3 is involved in several aspects of translation initiation; IF3 (together with IF1) helps in the dissociation of $70 \mathrm{~S}$ ribosomes into $30 \mathrm{~S}$ and 50 S subunits (Kaempfer 1972; Godefroy-Colburn et al. 1975), catalyzes the formation of 30 S initiation complexes (Wintermeyer and Gualerzi 1983), and selects the initiator tRNA against elongator tRNAs in those complexes (Hartz et al. 1989). The selection of the initiator tRNA by IF3 is based on the unique RNA part of the initiator tRNA (Risuleo et al. 1976), and, most likely, sequences located in the anticodon stem and loop (Hartz et al. 1989). The three GC base pairs of the anticodon stem adjacent to the loop of the initiator tRNA are important for initiation complex formation and in vitro translation (Seong and RajBhandary 1987), suggesting that this part of the anticodon stem might be important for recognition by IF3. Berkhout et al. (1986) showed that IF3 excludes initiator tRNA on a UUU codon, as though the initiation codon, itself, might be part of the recognition motif explored by IF3 during selection of the initiator tRNA.

We have examined those features of the initiator tRNA that are required for selection by IF3. Using toeprinting (Winter et al. 1987; Hartz et al. 1988, 1989; McPheeters et al. 1988; Blasi et al. 1989; Schaefer et al. 1989 | as the assay for tRNA binding in 305 initiation complexes, we tested initiator tRNA fragments and several mutant fragments for their ability to be selected by IF3 on various initiation codons.

'Corresponding author.

\section{Results}

To analyze $30 \mathrm{~S}$ complex formation on various translation initiation sites, we used the extension inhibition technique, also called toeprinting. cDNA synthesis on a template mRNA is terminated when the reverse transcriptase encounters a $30 \mathrm{~S}$ ribosomal subunit plus tRNA bound on the mRNA. The short cDNA is visualized as a toeprint band on a sequencing gel. The toeprint usually appears 15 bases downstream of the $A$ of the initiation codon if initiator tRNA is bound in the complex or at +15 from the first base of the cognate codon if elongator tRNA is bound. When compared to the band of the fulllength cDNA, the toeprint band is a quantitative measure of ternary complex formation (Hartz et al. 1989).

\section{Variations of A22 Met}

Using the toeprinting assay, we have shown previously that the synthetic initiator tRNA fragment A22 Met (Fig. 1) behaves very similarly to the whole tRNA $A_{f}^{\text {Met }}$ in $30 S$ initiation complex formation; A22 ${ }^{\text {Met }}$ is able to bind to the ribosomal $\mathrm{P}$ site in ternary complexes with $30 \mathrm{~S}$ subunits and T4 gene 32 in vitro mRNA /the pRS170 transcript) at the true translation initiation site. A22 Met is also selected over the elongators $t R N A^{C y s}$ and $t R N A^{\text {Phe }}$ by IF3 (Hartz et al. 1989). A22 Met binds to 30 S complexes with a lower affinity than the whole initiator tRNA.

To elucidate further the parts of the initiator tRNA that contribute to ternary complex formation and IF3 selection, we synthesized variants of A22 Met (Fig. 1). The fragments DA39 ${ }_{\mathrm{f}}^{\text {Met }}$ and AT42 Met contain an additional, unmodified $\mathrm{D}$ and $\mathrm{T} \Psi \mathrm{C}$ loop, respectively, whereas 


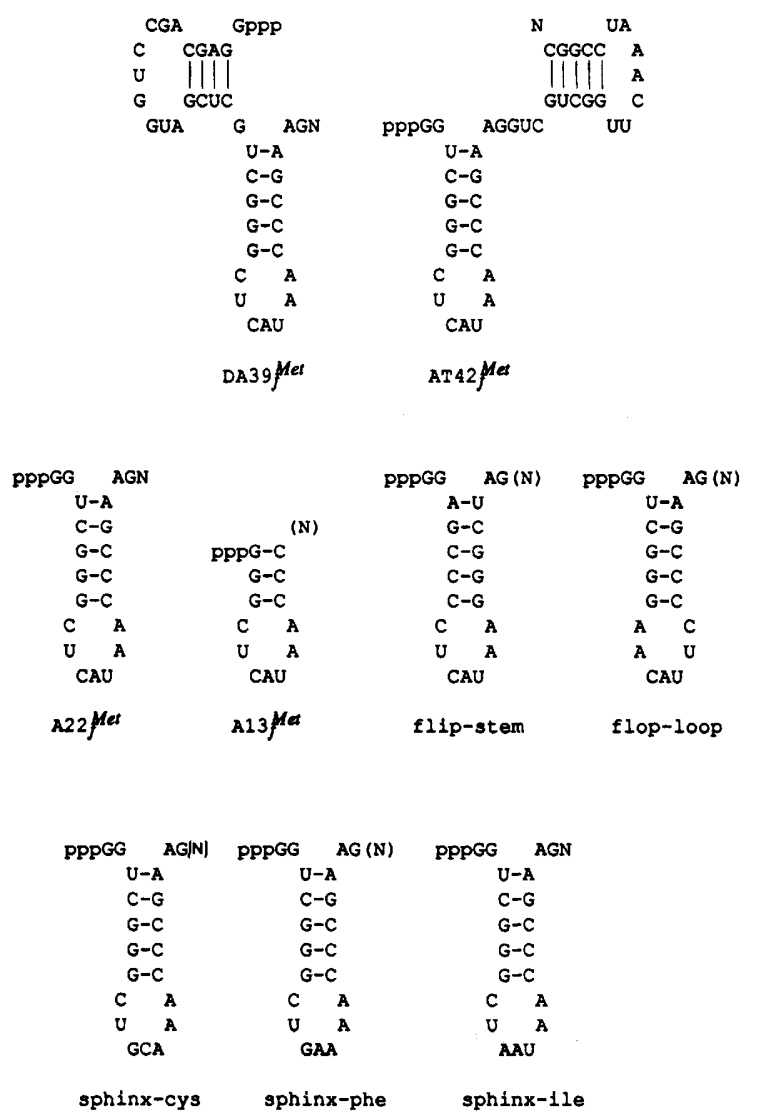

Figure 1. Synthetic tRNA fragments. Note the triphosphates at the $5^{\prime}$ ends and the extra random nucleotide $(\mathrm{N})$ on the $3^{\prime}$ ends. Some fragments (with $\mathrm{N}$ in parentheses) were also isolated without the $3^{\prime}$ random nucleotide.

$\mathrm{Al} 3 \mathrm{Met}$, as the shortest fragment, contains only the three GC base pairs of the anticodon stem. We further synthesized "flip-stem," with the base pairs of the anticodon stem switched, and "flop-loop," with the two 5' bases of the anticodon loop switched with the two $3^{\prime}$ bases of the loop. Finally, we synthesized anticodon variants with complementarity to UGU/UGC ("sphinx-cys"), UUU/UUC ("sphinx-phe"), and AUU ("sphinx-ile") codons. The fragments were tested for their ability to form ternary complexes with $30 \mathrm{~S}$ subunits and the pRS170 (gene 32) in vitro transcript in the toeprinting assay. All fragments, except flop-loop, yield strong toeprints 15 bases downstream from their respective codons (Fig. 2). Sphinx-cys and sphinx-phe recognize the UGU and UUU codons, respectively, as indicated by a corresponding shift of the toeprinting position. Sphinx-ile has no cognate codon on this mRNA (see below).

\section{Binding affinities}

The fragments of Figure 1 were titrated for their ability to form ternary complexes on the pRS170 transcript. At a constant $30 \mathrm{~S}$ subunit concentration, increasing amounts of tRNA fragments were added to toeprinting reactions (data not shown). The relative toeprints (toe- print stop $/ 5^{\prime}$ end + toeprint stop) were determined by radioscanning of the gels (Fig. 3). The relative toeprints do not reach $100 \%$ at apparently saturating concentrations of tRNA fragments because the reactions have not gone to completion during the $10-\mathrm{min}$ preincubation period. Reaction completion, at saturating concentrations of tRNA $\mathrm{f}_{\mathrm{f}}^{\text {Met }}$ and $\mathrm{A} 13_{\mathrm{f}}^{\text {Met }}$, takes $\sim 100 \mathrm{~min}$; at this time, relative toeprints of $100 \%$ are obtained with both $\mathrm{tRNA}_{\mathrm{f}}^{\mathrm{Met}}$ and $\mathrm{A} 13_{\mathrm{f}}^{\mathrm{Met}}$ (S. Ringquist, pers. comm.). Thus, the equilibrium is strongly toward ternary complex formation, and the number of complexes displaced by reverse transcriptase is negligible. The tRNA-binding step cannot be rate limiting because the reaction saturates as a function of tRNA concentration, even after $10 \mathrm{~min}$. The same is true for the mRNA-binding step (data not shown). The rate-limiting step is most likely the rearrangement of a preternary complex, in which both mRNA and tRNA are bound at the ribosome, but within which the tRNA anticodon is not base paired with the codon. The rearrangement creates a ternary complex in which the anticodon of the tRNA is base paired with a codon of the mRNA (Gualerzi and Pon 1981). Preternary complexes might equilibrate with tRNA, mRNA, and

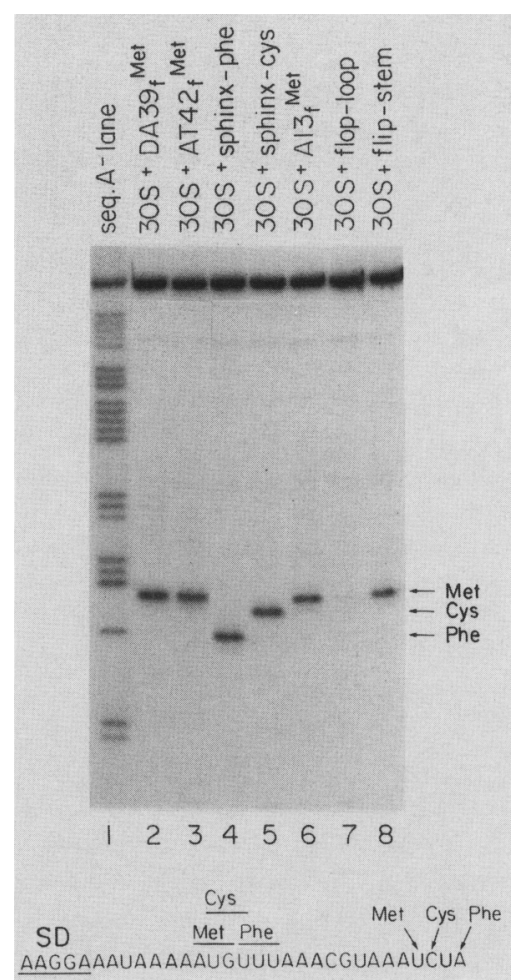

Figure 2. Detection of ternary complexes on the pRS170 transcript with several tRNA fragments. Reactions contained 0.2 $\mu \mathrm{M} 30 \mathrm{~S}$ subunits and $0.5 \mu \mathrm{M}$ of the fragments indicated above the lanes. Toeprint bands are marked with arrows. (Lane 1) A sequencing reaction containing ddTTP (A-lane). (Bottom) The location of the toeprints within the initiation domain of the pRS170 transcript, 15 nucleotides $3^{\prime}$ of the first base of the codons AUG, UGU, and UUU. The Shine and Dalgarno sequence $(S D)$ is underlined. 
Hartz et al.

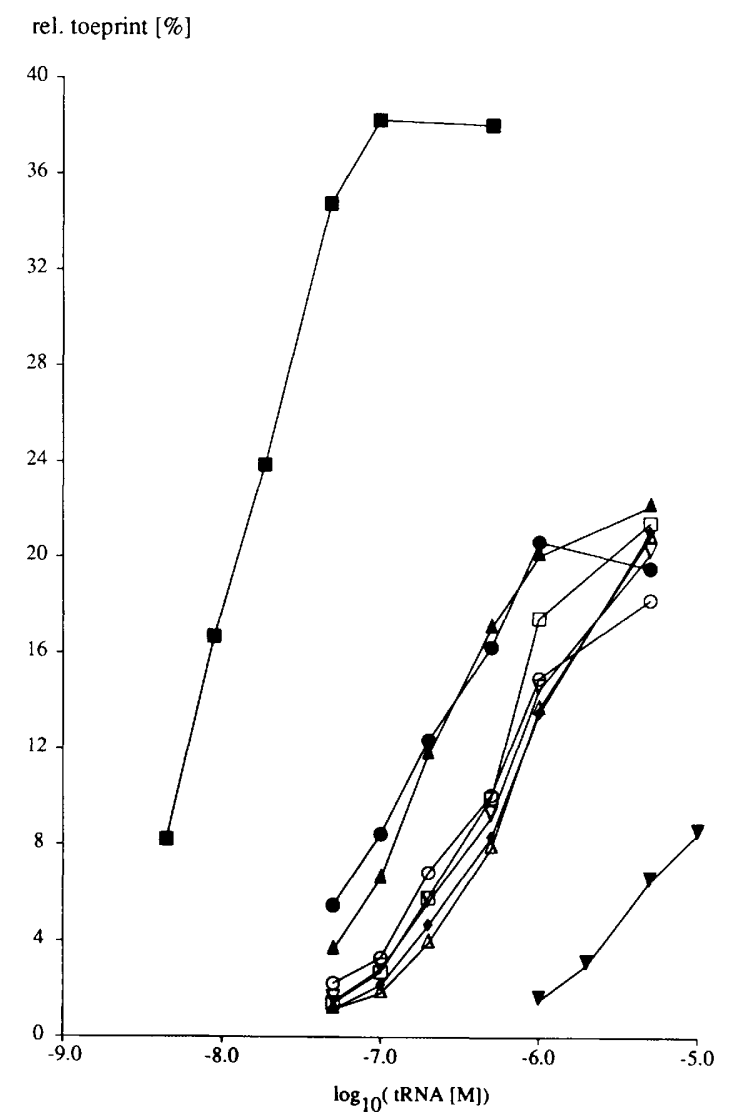

Figure 3. Dependence of ternary complex formation on the concentration of the synthetic tRNA fragments. Increasing amounts of the tRNA fragments AT42 Met, DA39Met, A13 Met, A22 Met, sphinx-cys, sphinx-phe, flip-stem, and flop-loop were added at various concentrations to reactions containing $0.2 \mu \mathrm{M}$ $30 S$ subunits and the pRS170 transcript. Relative toeprints of

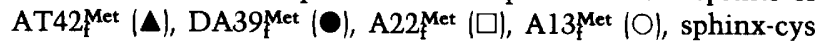
$(\nabla)$, sphinx-phe $(\Delta)$, flip-stem $(\Delta)$, and flop-loop $(\nabla)$ are plotted against the $\log _{10}$ of the fragment concentrations. Data for tRNAMet (D) are shown for comparison and are from Hartz et al. (1989). To determine the 5 ' ends only the 5 '-end band was measured by densitometric scanning with tRNA ${ }_{f}^{\text {Met, }}$, whereas all $5^{\prime}$ ends above the toeprint band were integrated in this experiment. This explains the apparent discrepancy in the relative toeprint intensity at saturation.

$30 S$ subunits quickly, whereas the speed of the rearrangement step limits the formation of the ternary complex (Gualerzi et al. 1977; Ellis and Conway 1984). The speed of ternary complex formation with tRNA Met $^{\text {and }}$ A13 $\mathrm{f}^{\mathrm{Met}}$ at saturating tRNA concentrations is practically identical and approximately linear over the 10-min preincubation period (data not shown). The apparently better binding of tRNA speeds of the rearrangement step with these tRNAs but probably reflects a higher binding affinity of tRNA int $^{\text {in }}$ the preternary complex. The binding of DA39met and AT42 Met appears to be slightly better than that of the fragments A13 ${ }_{\mathrm{f}}^{\text {Met }}, \mathrm{A} 22_{\mathrm{f}}^{\text {Met, }}$, sphinx-cys, sphinx-phe, and flip-stem, which all show very similar binding (Fig. 3). The binding of flop-loop was much poorer. Even the best binding fragment DA39Met still binds much worse than tRNA Met.

\section{Selection of initiator $t R N A$ fragments by IF3}

To test the ability of IF3 to select the synthetic initiator tRNA fragments, we devised competition experiments in which one AUG-reading fragment species was added together with the UGU- and UUU-reading fragments (sphinx-cys and sphinx-phe) in toeprinting reactions. The incubations contained $30 \mathrm{~S}$ subunits and the pRS170 transcript plus or minus IF3. The concentrations of the AUG-reading fragments were chosen so that they would yield a reasonable toeprint in competition with sphinxcys and sphinx-phe (Fig. 4, lanes 1, 4, 7, 10, 13, and 16). Addition of IF3 leads to a clear selection of the toeprint band generated by the AUG-reading fragment only if it is a "true" initiator tRNA fragment (Fig. 4). Even the smallest fragment $A 13_{\mathrm{f}}^{\text {Met }}$ is selected. The fragments flip-stem and flop-loop, with base changes in stem or loop, are not selected by IF3 over the fragments sphinxcys and sphinx-phe (Fig. 4, lanes 14, 15, 17, and 18). In those latter incubations, IF3 destabilizes all ternary complexes. Bases in the anticodon stem, loop, and the anticodon itself must be important for the selection of the initiator tRNA by IF3.

\section{Loop conformation of the initiator $t R N A$ fragments}

The anticodon loop conformation of several initiator tRNAs is different from that of the elongator tRNAs when the accessibility of loop phosphodiester bonds is probed with S1 nuclease (Wrede et al. 1979). The special loop conformation of the initiator tRNA (and its function as initiator tRNA) is dependent on the three GC base pairs of the anticodon stem (Seong and RajBhandary 1987). Here, we used the same technique to probe the anticodon loop conformation of several $3^{\prime}{ }^{32}$ pCp-labeled tRNA fragments. The positions of the $\mathrm{S} 1$ nuclease cleavages were determined on a gel by comparison with the alkaline hydrolysis ladder of the fragments, which itself was aligned with the sequence-specific cleavage pattern obtained with RNase $T_{1}$ and RNase $U_{2}$.

Flip-stem, flop-loop, sphinx-cys, and sphinx-phe all show S1 cuts in the loop region (Fig. 5). Cleavage positions are indicated in Figure 6. The cuts in the loop must occur more rapidly than cuts in the $3^{\prime}$ single-stranded region of the fragments to appear as labeled bands on the gel. The loops of the different fragments vary considerably in their sensitivity toward S1 nuclease, and so do the positions of the $S 1$ cuts. Especially prone to $S 1$ cleavage is the loop of the flop-loop fragment. The loop sequence of $\mathrm{A} 13 \mathrm{Met}$, however, is highly resistant to S1 cleavage. Only one potential cleavage site was detected (Fig. 6). Strong resistance to S1 nuclease was also shown by the loop region of $5^{\prime}{ }^{32} \mathrm{P}$-labeled A22 Met. No loop cleavages by nuclease $\mathrm{Sl}$ were observed under conditions in which the loops of $5^{\prime}{ }^{32} \mathrm{P}$-labeled flip-stem and sphinx-cys showed extensive S1 cuts (data not shown). It thus appears that true initiator tRNA fragments have a 


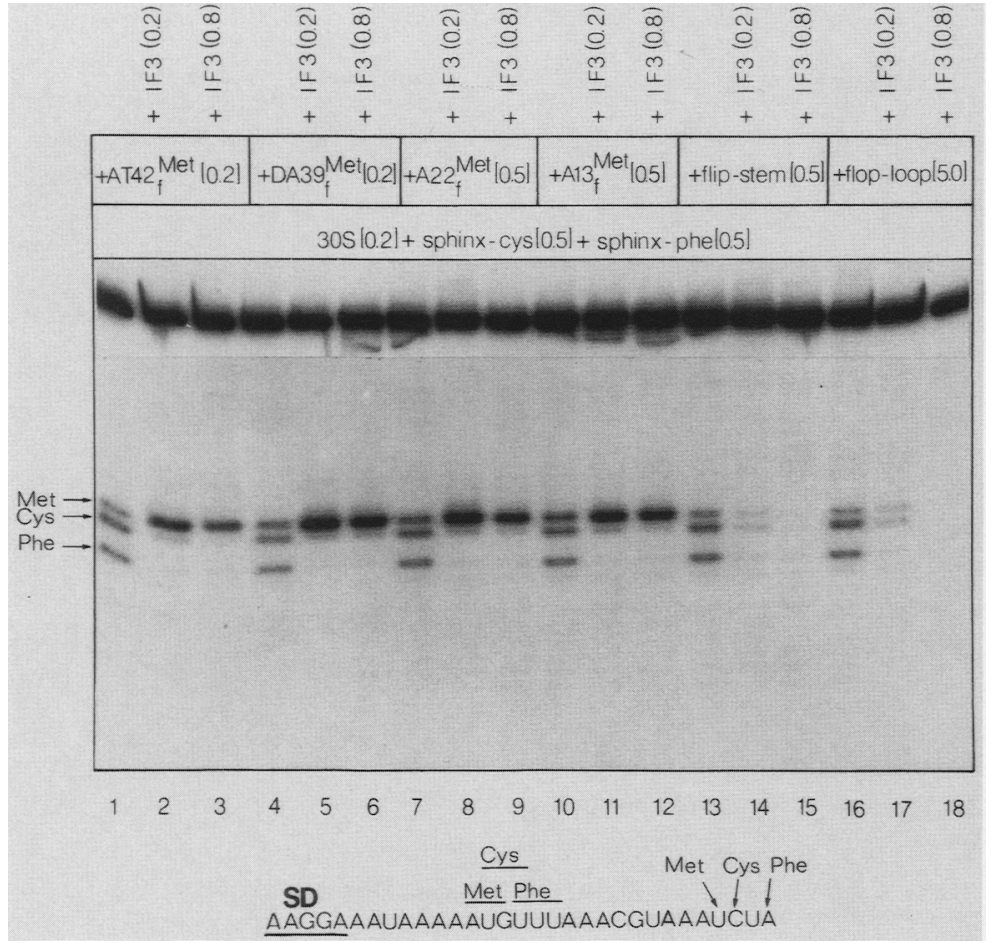

Figure 4. Selection of true tRNA AT42 Met, DA39Met, A22 Met, and A13 $3_{\mathrm{f}}^{\text {Met }}$ by IF3 against sphinx-cys and sphinx-phe on the pRS170 transcript. Reactions contained the components indicated above the lanes at the concentrations $(\mu \mathrm{M})$ indicated in parentheses. Toeprints are marked with arrows on the autoradiograph and in the sequence of the pRS170 transcript (bottom). different loop conformation than the tRNA fragments with base changes in anticodon stem, loop, or the anticodon.

\section{Selection of yeast and wheat germ initiator $t R N A$ by IF3}

The cytoplasmic initiator tRNAs of yeast and wheat germ (when charged with formylmethionine) are able to initiate translation properly in an E. coli in vitro system (Takeishi and Ukita 1968; Ghosh et al. 1974). Interestingly, their sequences differ extensively from that of $E$. coli initiator tRNA (Sprinzl et al. 1987); however, the three GC base pairs of the anticodon stem are conserved. The anticodon loops show differences mainly in nucleotide modifications (Fig. 7). We asked whether either eukaryotic initiator tRNA could be recognized by $E$. coli IF3 in our in vitro system. An equimolar mixture of each initiator tRNA with $E$. coli tRNA $^{\mathrm{Cys}}$ and tRNA ${ }^{\text {Phe }}$ produces toeprints at +15 from the cognate codons on the pRS170 in vitro transcript (Fig. 8). If IF3 is added in an equimolar amount to the $30 \mathrm{~S}$ subunits, the initiator tRNA toeprints prevail, whereas the elongator tRNA toeprints disappear. Clearly, yeast and wheat germ initiator tRNAs are selected by E. coli IF3. Most likely, the similar anticodon units of the initiator tRNAs are responsible for the selection (as suggested by the data in Fig. 4).

\section{Importance of the initiation codon}

In the experiments reported so far, we have identified parts of the initiator tRNA crucial for its selection by
IF3. The base pairing of the mRNA codon with the tRNA anticodon and the strength of that interaction might also play a role in initiator tRNA selection by IF3 (Berkhout et al. 1986). We tested the initiator tRNA fragment $\mathrm{Al} 3_{\mathrm{f}}^{\mathrm{Met}}$ on $\mathrm{mRNAs}$ with varied initiation codons (UUG, GUG, AUU) in competition with sphinxcys and sphinx-phe on the codons UGC and UUC, respectively. The synthetic mRNA SD8AUG (the T7 transcript of pT7SD8AUG) contains a UGC codon in the +1 reading frame (Fig. 9), which should allow for a CG base pair between the cytosine in the third position of the codon and the guanine in the sphinx-cys anticodon. A UGC codon is also present in the SD8UUG and SD8GUG mRNAs (Fig. 9). A13 ${ }_{\mathrm{f}}^{\text {Met }}$ was competed against sphinx-cys in 30S ternary complexes on those mRNAs. Two major toeprint stops at +15 from the AUG and UGC codons, respectively, were obtained (Fig. 9, lanes 1 and 7; Fig. 10, lane 4). Part of the lower reverse transcriptase stop is also contributed by $\mathrm{A} 13_{\mathrm{f}}^{\text {Met-containing }}$ complexes which, by themselves, yield a double toeprint with a strong +15 and a weaker +16 band (Fig. 10, lane 3). This makes the selection of $\mathrm{Al} 3_{\mathrm{f}}^{\text {Met }}$ against sphinxcys less obvious in $30 \mathrm{~S}$ complexes on SD8AUG, SD8UUG, and SD8GUG plus IF3 (Fig. 9, lanes 2, 3, 8, and 9; Fig. 10, lanes 5 and 6). Nevertheless, the control experiment with flip-stem and sphinx-cys plus IF3 clearly shows that neither sphinx-cys nor flip-stem are selected by IF3 because both toeprint bands disappear (Fig. 9, lanes 5, 6, 11, and 12). Thus, sphinx-cys is in no case selected by IF3, even when codon-anticodon basepairing with two GC base pairs can occur. On the other hand, A13 Met is selected by IF3 on the initiation codons UUG and GUG. Weak toeprint bands appear above the 


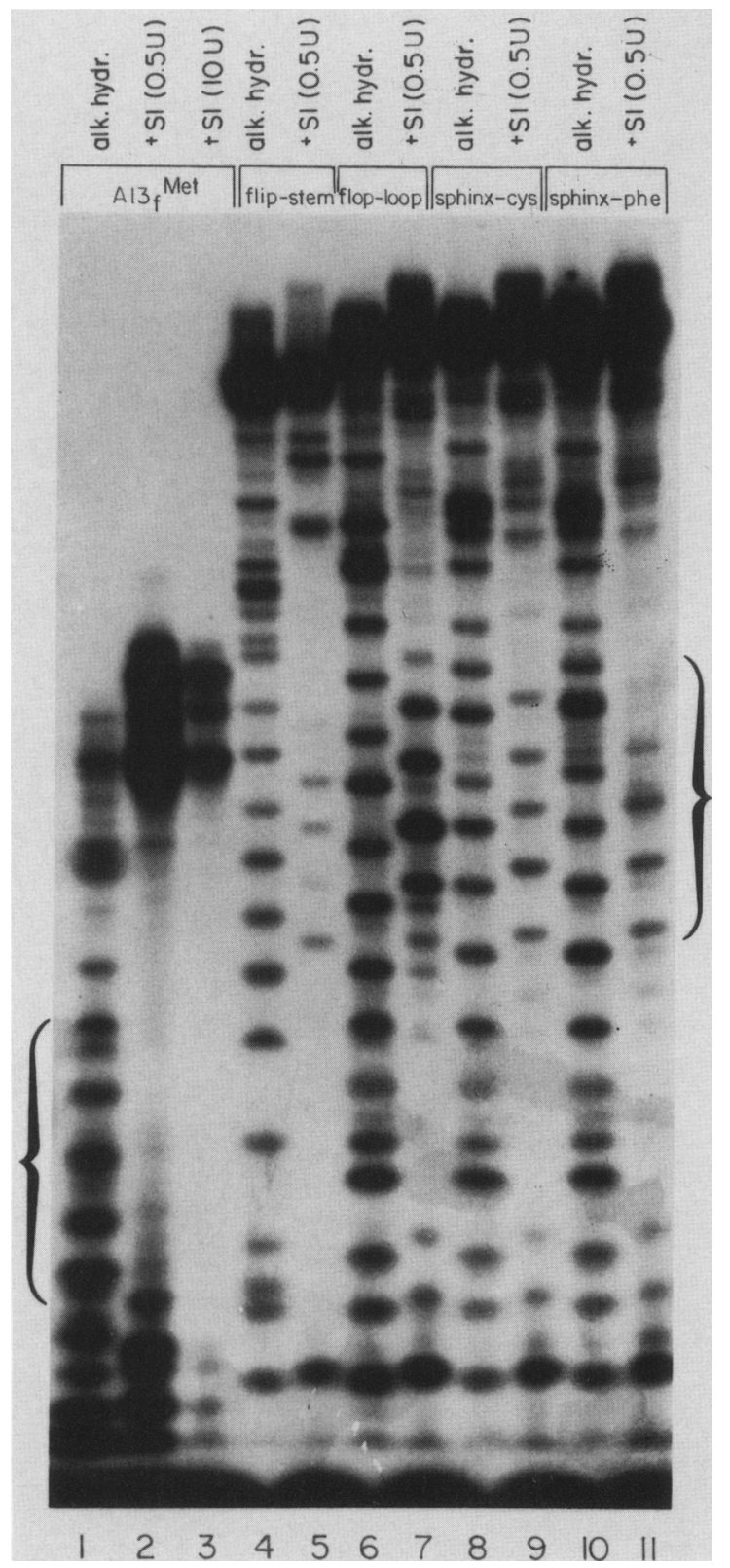

Figure 5. Probing of the loop conformation of ${ }^{32} \mathrm{pCp} 3$ '-endlabeled tRNA fragments with S1 nuclease. For each fragment, an alkaline hydrolysis ladder is shown at left and the S1 nuclease digest is shown at right (two lanes with different S1 nuclease concentrations for A13 right indicates the loop region of the fragments (except $\mathrm{Al} 13$ Met) where S1 nuclease cuts were found; the bracket on the left indicates the same loop region for A13 $3^{\text {Met. }}$

main stops, mainly at +11 and +12 from the AUG codon. They are not +15 from any cognate codon and are not understood.

We then investigated the consequences of a base change in the third position of the initiation codon for selection of initiator tRNA by IF3. The SD8AUU synthetic mRNA (with an AUU initiation codon) contains a UUC codon in the +1 reading frame, complementary to
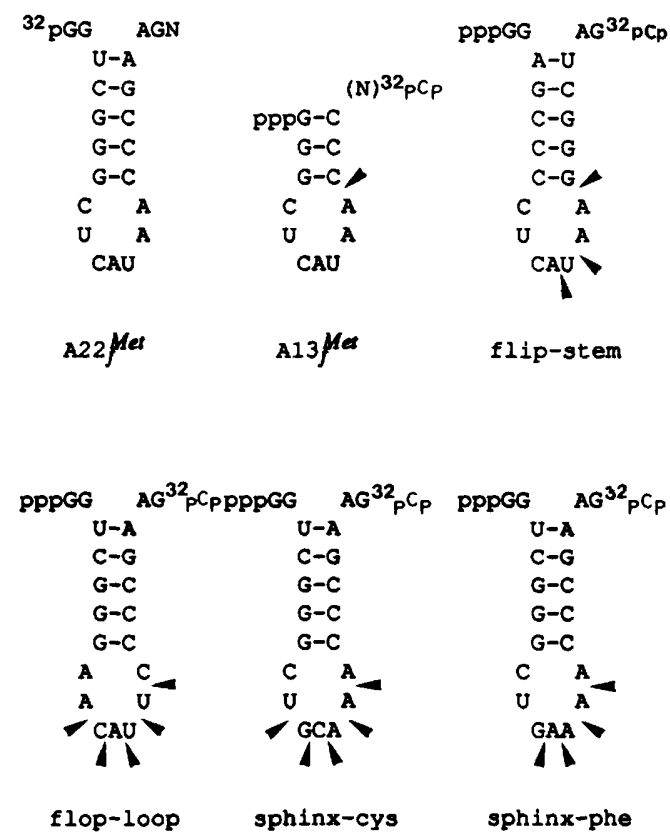

Figure 6. S1 nuclease cleavage sites. The positions of the cuts in the loop regions of $3^{\prime}{ }^{32} \mathrm{pCp}$-labeled synthetic tRNA fragments of Fig. 5 and of $5^{\prime}{ }^{32} \mathrm{P}$-labeled A2s $\mathrm{set}_{\text {(data not shown) are }}$ indicated with arrowheads.

the anticodon of sphinx-phe (Fig. 9). The AUU initiation codon is of particular interest, because so far it has only been found at the translational start site of infC, which encodes IF3 itself (Sacerdot et al. 1982). Models for autogenous regulation of IF3 translation have been proposed based on that unique initiation codon (Gold et al. 1984; Berkhout et al. 1986). A13 fet $^{\text {was }}$ added either alone or together with sphinx-phe in toeprinting reactions containing the SD8AUU mRNA. A13 $3_{\mathrm{fet}}^{\text {at high }}$ concentration yields a weak toeprint band at +15 and +12 from the AUU codon (Fig. 11, lane 3). Both bands disappear when IF3 is added in equimolar amount to the $30 S$ subunits (Fig. 11, lane 4). IF3 excludes A13 $3_{\mathrm{F}}^{\text {Met }}$ from the AUU codon. The same result was obtained when

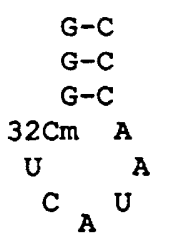

E.coli

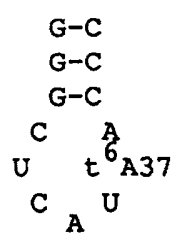

yeast

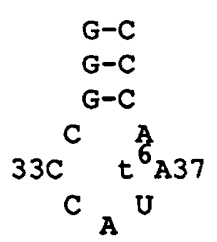

wheat germ
Figure 7. Comparison of the anticodon stem and loops of $E$. coli, yeast, and wheat germ initiator tRNAs. E. coli tRNAMet has $\mathrm{Cm}$ (2'-O-methylcytidine) instead of cytidine at position 32. Yeast and wheat germ initiator tRNAs, both have $\mathbf{t}^{6} \mathrm{~A}$ ( $N$-(\{9-3-D-ribofuranosylpurine-6-yl)carbamoyl)-threonine) instead of adenosine in position 37 . Furthermore, wheat germ initiator tRNA has a cytidine at position 33 instead of the "universal" uridine. The sequences are from Sprinzl et al. (1987). 


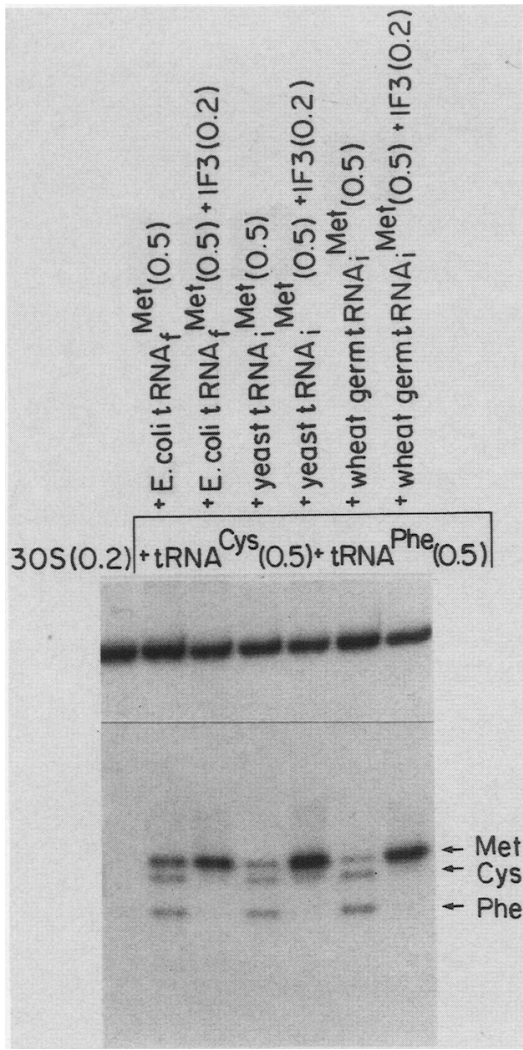

Figure 8. Selection of $E$. coli, yeast, and wheat germ initiator tRNAs by IF3 on the pRS170 transcript. The initiator tRNAs were competed against $E$. coli tRNA ${ }^{\text {Cys }}$ and tRNA ${ }^{\text {Phe }}$. Reactions contained the components indicated above the lanes at the concentrations $(\mu \mathrm{M})$ indicated in parentheses. Toeprints corresponding to ternary complexes with the three tRNAs are indicated by arrows.

A13 ${ }_{\mathrm{f}}^{\text {Met }}$ was competed with sphinx-phe. Toeprints originating from the respective complexes vanish in incubations containing IF3 (Fig. 11, lanes 6 and 7). The third base of the initiation codon must play a crucial role in the IF3 selection of initiator tRNA.

The AUU-reading fragment sphinx-ile (Fig. 1), which restores the base pair with the uridine in the third position of the AUU codon, was also tested in toeprinting reactions with SD8AUU mRNA, either alone or in competition with sphinx-phe. Signals from ternary complexes were obtained with both fragments at +15 from the cognate codons (Fig. 11, lane 9), but they disappeared in incubations including IF3 (Fig. 11, lanes 10 and 11).

\section{Discussion}

\section{IF3 selection of $t R N A$ fragments}

E. coli initiation factor IF3 is able to select initiator tRNA against elongator tRNAs in 30S ternary complexes (Hartz et al. 1989). A most striking new finding of this study is that only synthetic RNA fragments with the anticodon stem and loop sequences of initiator tRNA are selected by IF3. Three GC base pairs of the anticodon stem and a proper anticodon loop are sufficient for IF3 selection. Larger RNAs are not selected better by IF3 and show only modestly enhanced affinity for ribosomes during ternary complex formation. The flop-loop fragment shows much poorer binding than the other fragments. Weaker binding might be due to the two adenosines $5^{\prime}$ to the anticodon (Uhlenbeck et al. 1982). A13 ${ }_{\mathrm{f}}^{\text {Met }}$, with only three GC base pairs of the anticodon stem maintained [the minimum requirement for ternary complex formation is 2 bp (Dube et al. 1969;

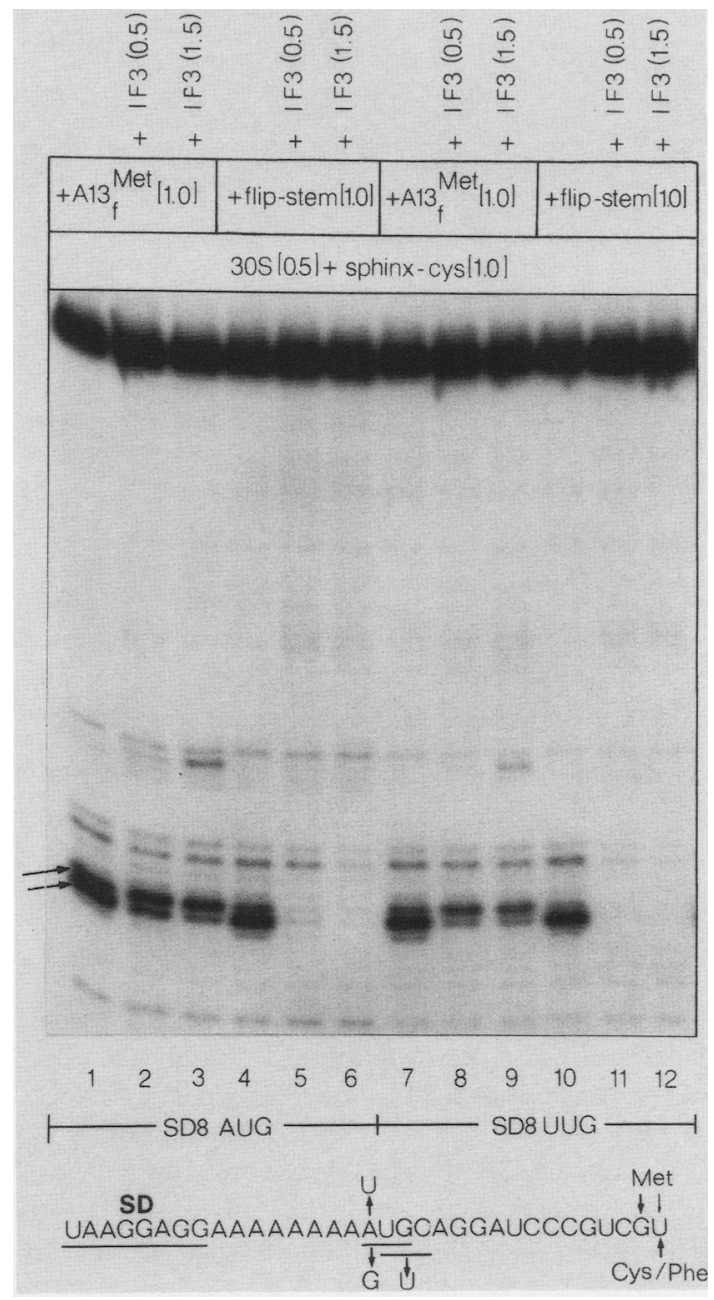

Figure 9. Selection of $\mathrm{A} 13_{\mathrm{f}}^{\text {Met }}$ by IF3 on an AUG and UUG initiation codon against sphinx-cys on a UGC codon. The left six lanes contained reactions with SD8AUG mRNA; the right six lanes contained reactions with SD8UUG mRNA. Besides A13 Met (lanes 1-3 and 7-9), flip-stem (lanes 4-6 and $10-12)$ was competed against sphinx-cys as a control experiment. Reactions contained all components indicated above the lanes at the concentrations $(\mu \mathrm{M})$ indicated in parentheses. Toeprints resulting from ternary complexes at +15 from the AUG and UGC codons are marked with arrows. Note that the upper toeprint band and part of the lower toeprint band are contributed by ternary complexes with A13 $3^{\text {Met }}$ and flip-stem. (Bottom) The initiation domain of SD8AUG mRNA is shown $3^{\prime}$ to the Shine and Dalgarno (SD) sequence with the changes in the initiation codon that yield SD8UUG, SD8GUG, and SD8AUU mRNA. 


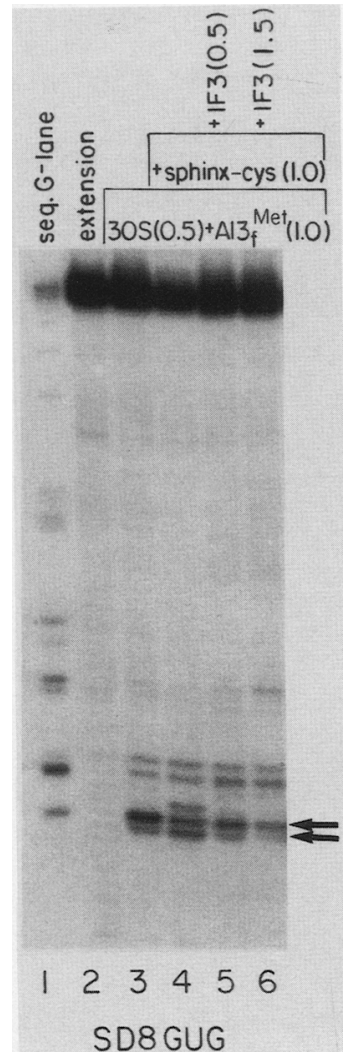

Figure 10. Selection of $\mathrm{A} 13^{\text {Met }}$ by IF 3 on a GUG initiation codon against sphinx-cys on a UGC codon. In addition to SD8GUG mRNA, reactions 3-6 contained the components indicated above the lanes at the concentrations $(\mu \mathrm{M})$ indicated in parentheses. Toeprints are indicated by arrows. Note that the upper band and part of the lower band are contributed by ternary complexes with A13 ${ }^{\text {Met }}$. (Lane 1) A sequencing reaction including ddCTP (G-lane).

Rose et al. 1983|], is selected by IF3. The three GC base pairs of $\mathrm{Al} 3_{\mathrm{i}}^{\mathrm{Met}}$ are conserved in all cytoplasmic initiator tRNAs (Sprinzl et al. 1987). Changing these base pairs in E. coli initiator tRNA hampers initiation complex formation and translation in vitro (Seong and RajBhandary 1987). In the fragment flip-stem, which IF3 fails to select, these GC base pairs are changed to CG base pairs. Thus, the GC base pairs of the anticodon stem are crucial for IF3 recognition of the initiator tRNA.

The three GC base pairs help to create a special conformation of the anticodon loop of the initiator tRNA, as measured by S1 nuclease cleavage (Wrede et al. 1979; Seong and RajBhandary 1987). This special loop conformation probably is one important feature for initiator tRNA selection by IF3. Strikingly, we find high resistance to $S 1$ nuclease in the anticodon loop of $\mathrm{A} 13_{\mathrm{fet}}^{\mathrm{Met}}$ and A22 Met but lower resistance in flip-stem. The same effect was observed previously by Seong and RajBhandary (1987), when they changed the three GC base pairs in the anticodon stem of initiator tRNA. Fragments not selected by IF3 (flip-stem, flop-loop, sphinx-cys, sphinxphe) all share a higher accessibility in their loops to $S 1$ cleavage and, thus, probably have different loop confor- mations. The requirement for a base in a specific position does not imply the recognition of that base by IF3; rather, the entire loop conformation could be a unit that is sensitive to base substitutions.

\section{Other initiator $t R N A s$}

Yeast and wheat germ initiator tRNAs are selected by IF3. Because the important features of the tRNA for IF3 selection are probably all located in the anticodon stem and loop, the minor differences in the anticodon loops of the initiator tRNAs (Fig. 7) do not interfere with IF3 selection. The anticodon loop conformations of $E$. coli, yeast, and mammalian initiator tRNA /which has the three GC base pairs of the anticodon stem and the same loop as wheat germ initiator tRNA) appear to be iden-

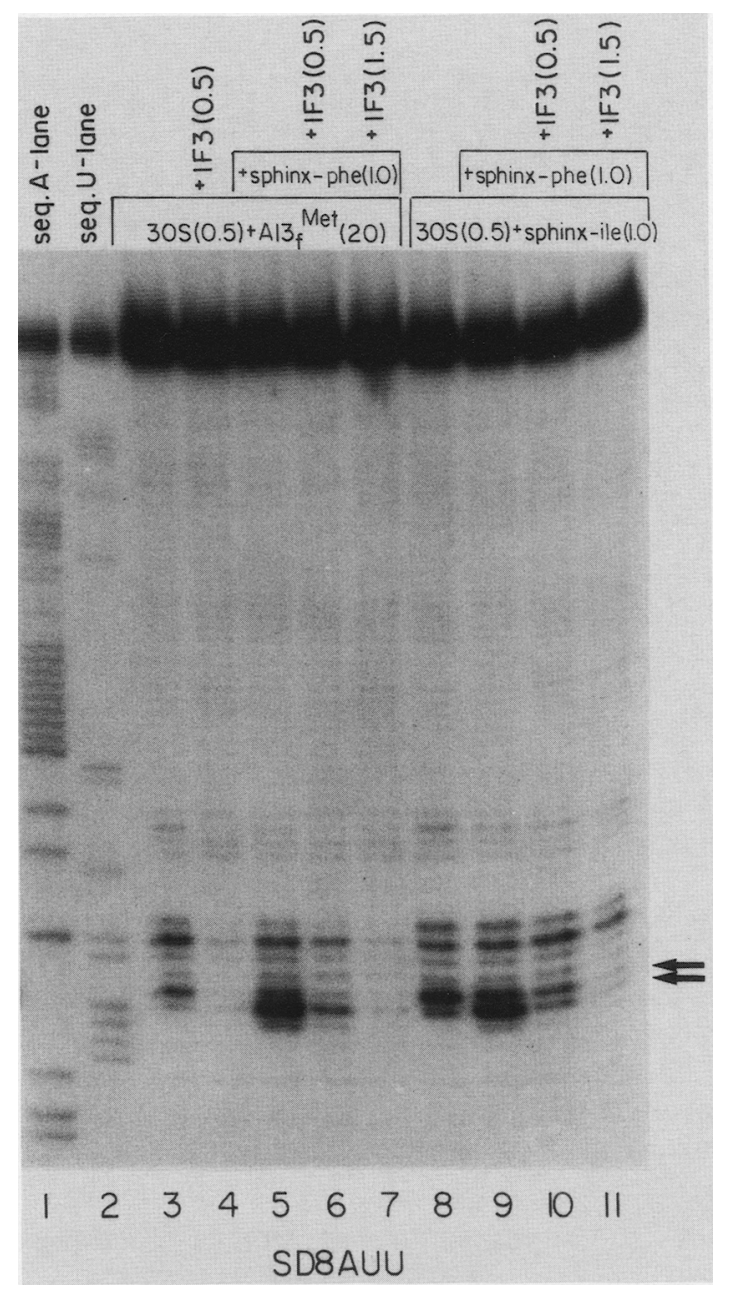

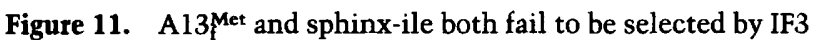
on the AUU initiation codon of SD8AUU mRNA. A13 $3_{\text {Met }}^{\text {(lanes }}$ 5-7) and sphinx-ile (lanes 9-11) were each competed against sphinx-phe, which forms ternary complexes on the UUC codon in the +1 frame. Lanes 3-11 contained the components above the lanes at the concentrations $(\mu \mathrm{M})$ indicated in parentheses. Toeprints at +15 from the cognate codons are indicated by arrows. (Lanes 1 and 2) Sequencing lanes containing ddTTP (Alane) and ddATP (U-lane), respectively. 
tical (Wrede et al. 1979). We speculate that those positions with varied nucleotides are not directly inspected by IF3 action but still allow the special anticodon loop conformation of initiator tRNAs.

What biological/biochemical function is served by the yeast initiator tRNA structure that responds to $E$. coli IF3? We see this result as implying the capacity in yeast to select the initiator tRNA as the sole occupant of the ribosomal P site during initiation, exactly as occurs in $E$. coli. Two wonderful papers from Donahue's lab (Cigan et al. 1988; Donahue et al. 1988) ask what requirements exist for initiation codon selection. The initiator tRNA anticodon was changed to $3^{\prime}-\mathrm{UCC}-5^{\prime}$, and that tRNA initiates at AGG codons following all the rules of translation initiation in yeast. Thus, yeast contains some mechanism for allowing an initiator to use codon/anticodon pairing to select the initiation codon, and that selection continues to operate with single nucleotide substitutions at the middle positions of the codon and anticodon (nucleotide substitutions that we have not tried yet for IF3 selectivity). As importantly, amino acid substitutions in the elF- $2 \beta$ protein create the dominant phenotype in yeast of allowing yeast initiator tRNA to start translation at a UUG codon (as occurs without difficulty in $E$. coli). We see Donahue's work as suggesting that a yeast initiation factor (perhaps even eIF-2 $\beta$ ) functions analogously to IF3, and probably the yeast initiation factor takes advantage of the same anticodon loop conformation that is sought by IF3. Sean Eddy (pers. comm.) asked whether eIF- $2 \beta$ from yeast has amino acid similarities with $E$. coli IF3, and he found none. Nevertheless, our data and Donahue's work suggest a functional conservation between $E$. coli and yeast: Probably ribosomes are themselves unable to strongly discriminate against elongator tRNAs, and both organisms use initiation factors and the special conformation of initiator tRNA anticodon loops to force initiation at an AUG using codon/anticodon pairing. Interestingly, yeast is but one amino acid substitution (in one gene, plus another mutation to cause overexpression of the methionine tRNA synthetase) away from being able to allow initiation at UUG codons, an interaction facilitated by IF3 (as in Fig. 9).

\section{Role of the initiation codon}

UUG and GUG serve as initiation codons for IF3 selection. The $5^{\prime}$ nucleotide of the initiation codon has no great influence on IF3 selection; thus, UUG and GUG initiation codons can be used quite efficiently as translational start sites in vivo (Gold 1988). CUG also serves as an initiation codon (Childs et al. 1985). In contrast, the third position of the initiation codon seems to be inspected by IF3 action. AUU gives poor toeprints with initiator tRNA fragments, and IF3 destabilizes them. Furthermore, in vivo translation from an AUA codon is very poor (Shinedling et al. 1987). The guanosine in the third position of the initiation codon is probably essential for IF3 selection of initiator tRNA.

The exclusion of initiator tRNA on the AUU initia- tion codon (the start codon of the IF3 mRNA) provides a mechanism for autogenous translational repression of IF3 expression. Such a mechanism was first proposed by Berkhout et al. (1986), based on the finding that IF3 excludes the initiator tRNA in 30S complexes on poly(U). Despite the exclusion of the initiator tRNA, IF3 is expressed at a reasonable level in the cell (Lestienne et al. 1982), and free 30S particles are probably saturated with IF3 (Howe and Hershey 1981).

The binding of IF3 to the 16S rRNA of 30S subunits near the 3' end of 16S rRNA (Wickstrom 1983), which must be in proximity to the ribosomal $\mathrm{P}$ site, makes it plausible that IF3 itself "inspects" the anticodon stem and loop of the tRNA bound in the ribosomal P site. This inspection must at least include the third position of the codon/anticodon interaction (Fig. 12). IF3 promotes the exchange of elongator tRNAs bound in ternary complexes in the ribosomal $\mathrm{P}$ site; elongator tRNAs are bound very stably in the absence of IF3 (Pon and Gualerzi 1974). Ternary complex formation with initiator tRNA is also catalyzed by IF3; initiator tRNA, however, is stably bound in the complex when IF3 is present (Risuleo et al. 1976). If IF3 lowers the activation energy for the codon/anticodon base-pairing step (Gualerzi et al. 1979|, it might bind to some part of the anticodon stem and loop of initiator tRNA to keep the initiator tRNA from being exchanged after ternary complex formation. Most likely, the third position of the initiation codon is part of such a binding site. Whereas only several bases need to be contacted directly, the other bases of the anticodon stem and loop are crucial to maintain certain bases in the conformation that can be bound by IF3.

\section{Methods}

\section{tRNA}

Total E. coli tRNA and uncharged $E$. coli tRNAMet and tRNA ${ }^{\text {Phe }}$ were purchased from Boehringer Mannheim. E. coli tRNA Cys was purchased from Subriden RNA. Yeast and wheat germ tRNA ${ }^{\text {Met }}$ were a gift of P. Sigler and R. Basavappa. The synthetic tRNA fragments shown in Figure 1 were generated by $T 7$ tran-

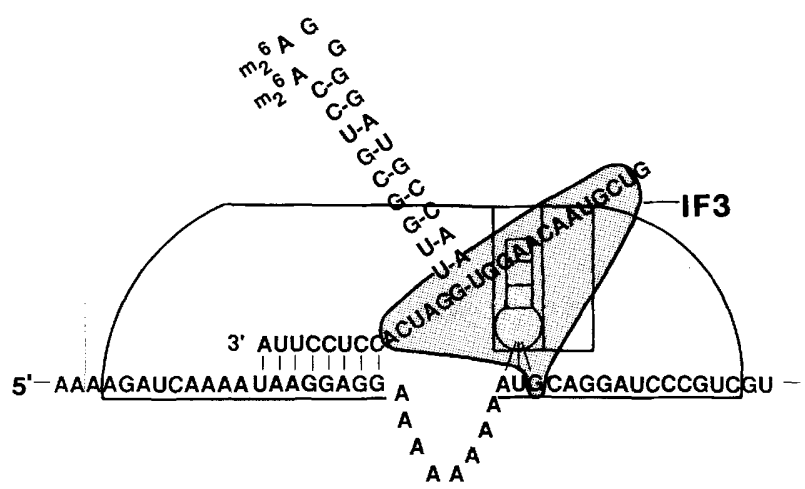

Figure 12. Model for IF3 selection of the initiator tRNA. IF3 binds to the $3^{\prime}$ end of $16 \mathrm{~S}$ rRNA and contacts some part of the anticodon stem and loop and the initiation codon. 
scription from the oligodeoxynucleotide pts as top strand and oligodeoxynucleotides as bottom strand, which contain the complementary sequence to the generated fragments (Table 1). T7 transcription was performed according to Milligan et al. (1987). The sphinx-ile fragment was synthesized by S. Beckmann. The tRNA fragments were purified on a $20 \%$ polyacrylamide gel. Some fragments were purified with and without an extra 3' nucleotide (indicated by $\mathrm{N}$ in parentheses in Fig. 1).

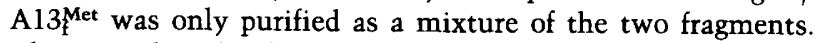
The $3^{\prime}$ nucleotide of purified tRNA fragments was determined by $3^{\prime}$-end labeling with ${ }^{32} \mathrm{pCp}$ according to Beckett and Uhlenbeck (1984), followed by RNase digestion and two-dimensional thin-layer chromatography (Nishimura 1972). Fragments without the extra 3 ' nucleotide exhibited the expected $3^{\prime}$ nucleotide, whereas fragments with an extra nucleotide were random (but with a preference for cytidine). Furthermore, $3^{\prime}$ end-labeled fragments were partially sequenced with RNase $T_{1}$ and RNase $\mathrm{U}_{2}$, according to Donis-Keller et al. (1977). The expected partial sequences were obtained.

\section{Enzymes}

AMV reverse transcriptase and MMLV reverse transcriptase were obtained from Life Sciences, Inc. and Bethesda Research Laboratories, respectively. T7 RNA polymerase was provided by O. Uhlenbeck. T4 polynucleotide kinase and T4 RNA ligase were purchased from New England Biolabs, Inc. Nuclease S1 and alkaline phosphatase were purchased from Boehringer Mannheim. RNase $T_{1}$ and RNase $U_{2}$ were obtained from Calbiochem.

\section{Initiation factor IF3 and Ribosomes}

Purified E. coli initiation factor IF3 was a gift from C. Gualerzi. $30 \mathrm{~S}$ subunits, prepared according to Kenney et al. (1979), were a gift from R. Traut.

\section{mRNAs}

In vitro RNAs from the PvulI-cut plasmids pRS170, pT7SD8AUG, pT7SD8UUG, pT7SD8GUG, and pT7SD8AUU were synthesized with $\mathrm{T} 7$ polymerase according to Lowary et al. (1986). The RNAs were purified on a $6 \%$ polyacrylamide gel.

\section{Plasmids}

The plasmid pRS170 contains the sequence from -92 to 107 of bacteriophage T4 gene 32 (Krisch and Allet 1982) and has been described in Hartz et al. (1989). To construct plasmids pT7SD8AUG, pT7SD8GUG, and pT7SD8UUG, the oligodeoxynucleotides shown in Table 1 were notch-cloned by D. Barrick into the large fragment of $B g I I I$ and PstI-digested $\mathrm{pBC} 39$ vector according to Childs et al. (1985). pBC39 was constructed by replacing the Pra2 promoter between EcoRI and HindIII of pBC29 (Childs et al. 1985) with the tacII promoter. The tacII promoter of the $\mathrm{pBC} 39$ clones was subsequently substituted by the T7 $\phi 10$ promoter to yield the plasmids pT7SD8AUG, pT7SD8UUG, and pT7SD8GUG. Plasmid pT7SD8AUU was constructed by cloning the two complementary oligodeoxynucleotides shown in Table 1 as double-stranded DNA into the large fragment of HindIII- and BamHI-digested plasmid pTS27. pTS27 is a derivative of pTS24 with the $F_{1}$ origin between SalIEcoRI cut out and replaced by a SalI-SmaI-EcoRI linker. pTS24 was constructed by replacing the EcoRI-HindIII Pra2 promoter fragment of pRS210 (McPheeters et al. 1988) with the phage T7 $\$ 10$ promoter (T. Schneider, unpubl.). Cloning protocols were adapted from Maniatis et al. (1982).

\section{Oligodeoxynucleotides}

Oligodeoxynucleotides were synthesized on an Applied Biosystems Model 380A DNA synthesizer and purified by preparative polyacrylamide gel electrophoresis. Oligodeoxynucleotides used as bottom strands to synthesize the tRNA fragments of Figure 1 were sequenced with AMV reverse transcriptase by using a protocol for RNA sequencing (McPheeters et al. 1986). The pts top strand oligodeoxynucleotide was used as primer. The sequences and use of the oligodeoxynucleotides are shown in Table 1.

\section{Extension inhibition}

Extension inhibition (or toeprinting) was performed essentially as described in Hartz et al. (1988). Ten-microliter toeprinting reactions contained $6.7 \mathrm{nM}$ of the in vitro transcripts, annealed to primer LP134 (Table 1), along with ribosomes, tRNAs, and IF3, as specified in the Results. Reactions with all ingredients were prepared on ice and preincubated for $10 \mathrm{~min}$ at $37^{\circ} \mathrm{C}$, fol-

Table 1. Description and use of oligodeoxynucleotides

\begin{tabular}{|c|c|}
\hline Sequence & Use \\
\hline taatacgactcactatag & pts top strand, $\mathrm{T} 7$ transcription \\
\hline ct tcgggt tatgagcccgacgagctaccaggctgctcctatagtgagtcgtatta & T7 transcription DA39Met \\
\hline gccggat t tgaaccgacgacct tcgggt tatgagcccgacctatagtgagtcgtat ta & T7 transcription AT42 Met \\
\hline cttagggttatgagcccgacctatagtgagtcgtat ta & T7 transcription A22 Met \\
\hline gggt tatgagccctatagtgagtcgtatta & $\mathrm{T} 7$ transcription $\mathrm{A} 13_{\mathrm{f}}^{\mathrm{Met}}$ \\
\hline ctagccet tatgaggggetcctatagtgagtcgtat ta & T7 transcription flip-stem \\
\hline ct tcggggaa tgt tcccgacctatagtgagtcgtat ta & T7 transcription flop-loop \\
\hline ct tcgggtt tgcagccegacctatagtgagtcgtat ta & T7 transcription sphinx-cys \\
\hline cttcgggtt tcagccegacctatagtgagtcgtatta & T7 transcription sphinx-phe \\
\hline ct tcgggt tat tagccegacctatagtgagtcgtatta & T7 transcription sphinx-ile \\
\hline gt tgggtaacgccaggg & LP134 primer, compl. to lacZ gene from 57 to 73 \\
\hline agct t tagatcaaaa taaggaggaaaaaaaaat tcag & cloning of pT7SD8AUU \\
\hline gatcctgaattetttectccttatt gatctaa & cloning of pT7SD8AUU \\
\hline gatcaaaa taaggaggaaaaaaaaatgca & cloning of pT7SD8AUG \\
\hline gatcaaaat aaggaggaaaaaaaagtgca & cloning of pT7SD8GUG \\
\hline gatcaaaataaggaggaaaaaaaattgca & cloning of pT7SD8UUG \\
\hline
\end{tabular}


lowed by primer extension with MMLV reverse transcriptase for $15 \mathrm{~min}$ at $37^{\circ} \mathrm{C}$.

\section{Labeling of tRNA fragments}

tRNA fragments were $3^{\prime}$-end-labeled with ${ }^{32} \mathrm{pCp}$ according to Beckett and Uhlenbeck (1984). With the exception of A13 only fragments without the extra random nucleotide on the $3^{\prime}$ end were $3^{\prime}$-end-labeled. To 5' ${ }^{32}$ P-end-labeled A22Met, flipstem, and sphinx-cys, 10 pmoles of each fragment was dephosphorylated with 1 unit calf intestinal alkaline phosphatase in a $50-\mu l$ reaction containing $50 \mathrm{~mm}$ Tris $-\mathrm{Cl}(\mathrm{pH} 8.0)$ and $0.1 \mathrm{~mm}$ EDTA. The reaction was incubated for $30 \mathrm{~min}$ at $45^{\circ} \mathrm{C}$. The samples were phenol extracted four times, and the fragments were precipitated with three volumes of ethanol. 5' 32P-labeling was performed with $\left[\gamma^{-32} \mathrm{P}\right] \mathrm{ATP}$, using a standard protocol for oligodeoxynucleotide labeling (Maniatis et al. 1982). Only fragments with an extra random nucleotide on the $3^{\prime}$ end were $5^{\prime}$. end-labeled.

\section{Partial alkaline hydrolysis of $t R N A$ fragments}

Four-microliter reactions contained 1.6 pmoles of $3^{\prime}$ - or $5^{\prime}$-endlabeled fragments in $50 \mathrm{~mm}$ sodium carbonate buffer (pH 9.0), 1 mM EDTA. They were incubated for $8 \mathrm{~min}$ at $95^{\circ} \mathrm{C}$ and cooled, and $10 \mu \mathrm{l}$ of loading buffer was added. Five-microliter samples were analyzed on a $20 \%$ polyacrylamide gel containing $8 \mathrm{M}$ urea.

\section{Probing of anticodon loop conformation with nuclease S1}

Nuclease S1 digestions were performed essentially as described by Wrede et al. (1979). Five-microliter reactions contained 0.8 pmoles of $3^{\prime}$ - or $5^{\prime}$-end-labeled fragments and $1 \mu \mathrm{g}$ of carrier tRNA in $25 \mathrm{~mm}$ sodium acetate (pH 4.5), $5 \mathrm{~mm} \mathrm{MgCl}_{2}, 50 \mathrm{mM}$ $\mathrm{KCl}$, and $1 \mathrm{mM} \mathrm{ZnCl}_{2}$. Nuclease $\mathrm{S} 1$ was added at the concentrations indicated in the figure legends, and the reactions were incubated for various time intervals at $37^{\circ} \mathrm{C}$. Only reactions from 10-min incubations, which showed the most extensive loop cleavage, are shown in Figure 5. Reactions were stopped by adding $10 \mu \mathrm{l}$ of loading dye (Hartz et al. 1988) containing $4 \mathrm{~mm}$ ATP and $0.2 \mu \mathrm{g} / \mu \mathrm{l}$ carrier tRNA. Five-microliter samples were analyzed on a $20 \%$ polyacrylamide gel containing $8 \mathrm{M}$ urea. Cleaved RNAs that carry the $3^{\prime}$-end label and are created by S1 digestion (with $5^{\prime}$-phosphate ends) should migrate faster than those created by alkaline hydrolysis [with $5^{\prime}$ hydroxyl ends (Wrede et al. 1979)|. However, in our experiments, S1-cleaved fragments appear to run slightly slower than alkaline-cleaved fragments; the dinucleotides cleaved off in the $3^{\prime}$ singlestranded region appear to migrate slightly slower than the dinucleotides created by alkaline hydrolysis, and even the fulllength fragments appear to run slower in the lanes with S1 incubation (Fig. 5). This effect of S1 is not observed with 5 '-end-labeled fragments (data not shown). If the S1 preparation contained a 3 ' phosphatase as a contaminant, only the electrophoresis of 3'-end-labeled fragments would be affected. We assigned the S1 cleavage sites based on these observations.

\section{Relative toeprints}

To quantify the toeprint stop and the 5 -end stops, the gels were scanned on an AMBIS Systems radioanalytic imaging system and the bands were electronically integrated. Relative toeprints (\%) were calculated: (toeprint/toeprint $+5^{\prime}$ ends) $\times 100$. To quantify the $5^{\prime}$ ends, all bands $5^{\prime}$ of the toeprint band were integrated.

\section{Acknowledgments}

We thank $R$. Traut for providing 30 S ribosomal subunits and $P$. Sigler and R. Basavappa for supplying yeast and wheat germ initiator tRNAs. We also thank D. McPheeters and S. Beckmann for the preparation of the tRNA fragments A22 Met and sphinxile. We are indebted to $C$. Gualerzi for his generous gift of large quantities of purified E. coli initiation factor IF3. We are also grateful to D. Irvine for helpful discussions. This work was supported by National Institutes of Health research grant GM-28685.

The publication costs of this article were defrayed in part by payment of page charges. This article must therefore be hereby marked "advertisement" in accordance with 18 USC section 1734 solely to indicate this fact.

\section{References}

Beckett, D. and O.C. Uhlenbeck. 1984. Enzymatic synthesis of oligoribonucleotides. In Oligonucleotide synthesis a practical approach (ed. M.J. Gait), pp. 185-197. (IRL Press, Oxford.

Berkhout, B., C.J. Van der Laken, and P.H. Van Knippenberg. 1986. Formylmethionyl-tRNA binding to $30 \mathrm{~S}$ ribosomes programmed with homopolynucleotides and the effect of translational initiation factor 3. Biochim. Biophys. Acta 866: $144-153$.

Blasi, U., K. Nam, D. Hartz, L. Gold, and R. Young. 1989. Dual translational initiation sites control function of lambda $\mathrm{S}$ gene. EMBO I. 8: 3501-3510.

Childs, J., K. Villanueba, D. Barrick, T.D. Schneider, G. Stormo, L. Gold, M. Leitner, and M. Caruthers. 1985. Ribosome binding site sequences and function. In Sequence Specificity in Transcription and Translation (ed. R. Calendar and L. Gold), pp. 341-350. Alan R. Liss, New York.

Cigan, A.M., L. Feng, and T.F. Donahue. 1988. tRNA Met func- $^{\text {Met }}$ tions in directing the scanning ribosome to the start site of translation. Science 242: 93-97.

Donahue, T.F., A.M. Cigan, E.K. Pabich, and B.C. Valavicius. 1988. Mutations at a $\mathrm{Zn}$ (II) finger motif in the yeast eIF-2.ft3 gene after ribosomal start-site selection during the scanning process. Cell 54: 621-632.

Donis-Keller, H., A.M. Maxam, and W. Gilbert. 1977. Mapping adenines, guanines and pyrimidines in RNA. Nucleic Acids Res. 4: 2527-2538.

Dube, S.K., P.S. Rudland, B.F.C. Clark, and K.A. Marcker. 1969. A structural requirement for codon-anticodon interaction on the ribosome. Cold Spring Harbor Symp. Quant. Biol. 34: $161-166$.

Ellis, S. and T.W. Conway. 1984. Initial velocity kinetic analysis of $30 S$ initiation complex formation in an in vitro system derived from Escherichia coli. I. Biol. Chem. 259: 7607-7614.

Ghosh, K., P. Ghosh, M. Simsek, and U. RajBhandary. 1974. Initiator methionine transfer ribonucleic acid from wheat embryo. Purification, properties, and partial nucleotide sequences. J. Biol. Chem. 249: 4720-4729.

Godefroy-Colburn, T., A.D. Wolfe, J. Dondon, M. GrunbergManago, P. Dessen, and D. Pantaloni. 1975. Light-scattering studies showing the effect of initiation factors on the reversible dissociation of Escherichia coli ribosomes. J. Mol. Biol. 94: 461-478.

Gold, L. 1988. Post-transcriptional regulatory mechanisms in $E$. coli. Annu. Rev. Biochem. 57: 199-233.

Gold, L., G. Stormo, and R. Saunders. 1984. Escherichia coli translational initiation factor IF3: A unique case of transla- 
tional regulation. Proc. Natl. Acad. Sci. 81: 7061-7065.

Gualerzi, C. and C.L. Pon. 1981. Protein biosynthesis in procaryotic cells: Mechanism of $30 \mathrm{~S}$ initiation complex formation in Escherichia coli. In Structural aspects of recognition and assembly in biological macromolecules (ed. L. Balaban, J.L. Sussman, W. Traub, and A. Yonath), pp. 805-826. Balaban, M. ISS, Rehovot.

Gualerzi, C., G. Risuleo, and C.L. Pon. 1977. Initial rate kinetic analysis of the mechanism of initiation complex formation and the role of initiation factor IF-3. Biochemistry 16: $1684-1689$.

1979. Mechanism of the spontaneous and initiation factor 3-induced dissociation of $30 \mathrm{~S}$ aminoacyl-tRNA polynucleotide ternary complexes. $J$. Biol. Chem. 254: 44-49.

Hartz, D., D.S. McPheeters, R. Traut, and L. Gold. 1988. Extension inhibition analysis of translation initiation complexes. Methods Enzymol. 164: 419-425.

Hartz, D., D.S. McPheeters, and L. Gold. 1989. Selection of the initiator tRNA by Escherichia coli initiation factors. Genes Dev. 3: 1899-1912.

Howe, J.G. and J.W.B. Hershey. 1981. A sensitive immunoblotting method for measuring protein synthesis initiation factor levels in lysates of Escherichia coli. I. Biol. Chem. 256: $12836-12839$.

Kaempfer, R. 1972. Initiation factor IF-3: A specific inhibitor of ribosomal subunit association. J. Mol. Biol. 71: 583-598.

Kenney, J.W., T.G. Fanning, J.M. Lambert, and R.R. Traut. 1979. The subunit interphase of the Escherichia coli ribosome. Crosslinking of $30 \mathrm{~S}$ protein S9 to proteins of the $50 \mathrm{~S}$ subunit. J. Mol. Biol. 135: 151-170.

Krisch, H. and B. Allet. 1982. Nucleotide sequences involved in bacteriophage T4 gene 32 translational self-regulation. Proc. Natl. Acad. Sci. 79: 4937-4941.

Lestienne, P., J. Dondon, J.A. Plumbridge, J.G. Howe, J. Mayaux, M. Springer, S. Blanquet, J.W.B. Hershey, and M. Grunberg-Manago. 1982. Expression of the gene for Escherichia coli initiation factor IF-3 in vivo and in vitro. Overproduction in recombinant-plasmid-bearing strains. Eur. I. Biochem. 123: 483-488.

Lowary, P., J. Sampson, J. Milligan, D. Groebe, and O.C. Uhlenbeck. 1986. A better way to make RNA for physical studies. In Structure and dynamics of RNA (ed. P.H. van Knippenberg and C.W. Hilbers), pp. 69-76. Plenum Press, New York.

Maniatis, T., E.F. Fritsch, and J. Sambrook. 1982. Molecular cloning: A laboratory manual. Cold Spring Harbor Laboratory Press, Cold Spring Harbor, New York.

McPheeters, D.S., G.D. Stormo, and L. Gold. 1988. The autogenous regulatory site on the bacteriophage T4 gene 32 messenger RNA. I. Mol. Biol. 201: 517-535.

McPheeters, D.S., A. Christensen, E.T. Young, G. Stormo, and I. Gold. 1986. Lysozyme gene. Nucleic Acids Res. 14: 58135826.

Milligan, J.F., D.R. Groebe, G.W. Witherell, and O.C. Uhlenbeck. 1987. Oligoribonucleotide synthesis using T7 RNA polymerase and synthetic DNA templates. Nucleic Acids Res. 15: 8783-8798.

Nishimura, S. 1972. Minor components in transfer RNA: Their characterization, location, and function. Prog. Nucleic Acids Res. Mol. Biol. 12: 49-85.

Pon, C.L. and C. Gualerzi. 1974. Effect of initiation factor 3 binding on the $30 \mathrm{~S}$ ribosomal subunits of Escherichia coli. Proc. Natl. Acad. Sci. 71: 4950-4954.

Risuleo, G., C. Gualerzi, and C. Pon. 1976. Specificity and properties of the destabilization, induced by initiation factor IF-3, of ternary complexes of the 30-S ribosomal subunit,
aminoacyl-tRNA and polynucleotides. Eur. I. Biochem. 67: 603-613.

Rose, S.J. III, P.T. Lowary, and O.C. Uhlenbeck. 1983. Binding of yeast tRNA ${ }^{\text {Phe }}$ anticodon arm to Escherichia coli $30 \mathrm{~S}$ ribosomes. J. Mol. Biol. 167: 103-117.

Sacerdot, C., G. Fayat, P. Dessen, M. Springer, J.A. Plumbridge, M. Grunberg-Manago, and S. Blanquet. 1982. Sequence of a $1.26 \mathrm{~kb}$ DNA fragment containing the structural gene for $\mathrm{E}$. coli initiation factor IF3: Presence of an AUU initiator codon. EMBO I. 1: 311-315.

Schaefer, E.M., D. Hartz, L. Gold, and R.D. Simoni. 1989. Ribosome binding sites and RNA processing sites in the transcript of the unc operon of Escherichia coli. J. Bacteriol. 171: 3901-3908.

Seong, B.L. and U.L. RajBhandary. 1987. Escherichia coli formylmethionine tRNA: Mutations in ${ }_{C}{ }^{G}{ }_{C}{ }_{C}$ sequence conserved in anticodon stem of initiator tRNAs affect initiation of protein synthesis and conformation of anticodon loop. Proc. Natl. Acad. Sci. 84: 334-338.

Shinedling, S., M. Gayle, D. Pribnow, and L. Gold. 1987. Mutations affecting translation of the bacteriophage T4 rIIB gene cloned in Escherichia coli. Mol. Gen. Genet. 207: 224-232.

Sprinzl, M., T. Hartmann, F. Meissner, J. Moll, and T. Vorderwulbecke. 1987. Compilation of tRNA sequences and sequences of tRNA genes. Nucleic Acids Res. Suppl. 15: r53r188.

Takeishi, K. and T. Ukita. 1968. Characterization of two species of methionine transfer ribonucleic acid from bakers' yeast. J. Biol. Chem. 243: 5761-5769.

Uhlenbeck, O.C., P.T. Lowary, and O.L. Wittenberg. 1982. Role of the constant uridine in binding of yeast tRNA ${ }^{\text {Phe }}$ anticodon arm to 30 S ribosomes. Nucleic Acids Res. 10: 33403352.

Wickstrom, E. 1983. Nuclease mapping of the secondary structure of the 49-nucleotide 3 ' terminal cloacin fragment of Escherichia coli 16S RNA and its interactions with initiation factor 3. Nucleic Acids Res. 11: 2035-2052.

Winter, R.B., L. Morrissey, P. Gauss, L. Gold, T. Hsu, and J. Karam. 1987. Bacteriophage T4 regA protein binds to mRNAs and prevents translation initiation. Proc. Natl. Acad. Sci. 84: 7822-7826.

Wintermeyer, W. and C. Gualerzi. 1983. Effect of Escherichia coli initiation factors on the kinetics of N-AcPhe-tRNA ${ }^{\text {Phe }}$ binding to $30 \mathrm{~S}$ ribosomal subunits. A fluorescence stoppedflow study. Biochemistry 22: 690-694.

Wrede, P., N. Woo, and A. Rich. 1979. Initiator tRNAs have a unique anticodon loop conformation. Proc. Natl. Acad. Sci. 76: 3289-3293. 


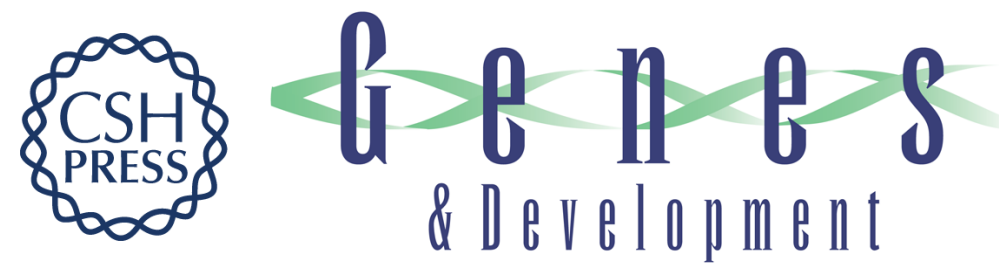

\section{Domains of initiator tRNA and initiation codon crucial for initiator tRNA selection by Escherichia coli IF3.}

D Hartz, J Binkley, T Hollingsworth, et al.

Genes Dev. 1990, 4:

Access the most recent version at doi:10.1101/gad.4.10.1790

References This article cites 38 articles, 15 of which can be accessed free at:

http://genesdev.cshlp.org/content/4/10/1790.full.html\#ref-list-1

License

Email Alerting

Service

Receive free email alerts when new articles cite this article - sign up in the box at the top right corner of the article or click here.

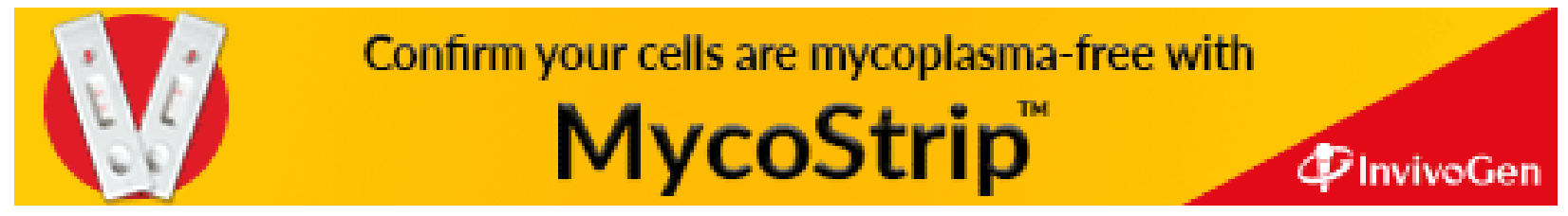

